\title{
Configuration method of PSS lead-lag compensator parameters
}

\author{
Yingfeng Zhu' ${ }^{1 *}$, Yongming Zhang ${ }^{2}$, Xiaosu Xie ${ }^{1}$, Dong Yang ${ }^{1}$, Song Gao ${ }^{1}$, Weichao Zhang ${ }^{1}$, Xunkui Yuan ${ }^{1}$ \\ ${ }^{1}$ State Grid Shandong Electric Power Research Institute, JiNan, 250003, China \\ ${ }^{2}$ Shan Dong Zhong Shi Yi Tong Group Limited Corporation, JiNan, 250003, China
}

\begin{abstract}
Power System Stabilizer (PSS) is an important measure to increase damping and suppress low frequency oscillations of power system. During PSS test, the time constant of lead-lag compensator need to be configured, considering the inefficiency and optimization problem when configuring parameters empirically, this paper proposes automatic configuration method of PSS parameters based on Matlab, by setting the expected phase value at each frequency and constructing the objective function with the sum of phase differences square, the automatic calculation of PSS parameters under specific phase requirements at each frequency can be realized, the detailed steps of this method are given, and the method is verified during the PSS test of an $300 \mathrm{Mw}$ thermal-power generating unit finally.
\end{abstract}

\section{Introduction}

Low-frequency oscillation largely affects the stable operation of the power system. The main reason of lowfrequency oscillation are negative-damping mechanism ${ }^{[1]}$, forced-oscillation mechanism ${ }^{[2]}$, nonlinear-mechanism ${ }^{[3]}$ and strong-resonance mechanism ${ }^{[4]}$, the negativedamping mechanism is a classic theory when studying low-frequency oscillation. Due to the phase-lag characteristics of the automatic regulator, excitation system and generator rotor winding, negative-damping torque is produced, which bring negative-damping of power system. For this reason, the power system stabilizer (PSS) is usually added to the regulator. PSS is an additional control device that adjusts the output rotor voltage with automatic-regulator to achieve the purpose of damping power system and other oscillations.

Currently, PSS2B model is widely used in the world. The PSS2B model has two input signals: speed and electric power. Only by correctly configuring the leadlag time constant of the PSS2B model, can PSS have its due effect. At present, EXCEL table is often used to adjust the time constant during PSS test, which takes a lot of time and can not get the best effect. This paper proposes an automatic calculation method based on Matlab, and the new method is verified during PSS test.

\section{Introduction of PSS2B Model}

\subsection{The Transfer Function of PSS2B Model}

PSS2B model's transfer function is shown in Figure 1. $\omega$ is signal of generator's speed, Pe is signal of generator's power, $\mathrm{T} \omega 1 \sim \mathrm{T} \omega 4$ is time constants of blocking compensator, $\mathrm{T}_{1} \sim \mathrm{T}_{5}$ and $\mathrm{T}_{10}$ are time constants of leadlag compensator (two or three-order compensation compensator may be used), $\mathrm{T}_{6} \mathrm{~T}_{7}$ are inertial time constants, $T_{8}, T_{9}$ are time constants of torsional vibration filter, $\mathrm{T}_{11} \sim \mathrm{T}_{14}$ are lead-lag time constants of automatic voltage regulator, $\mathrm{M}, \mathrm{N}$ are the orders of torsional vibration filter, $\mathrm{P}_{\mathrm{M}}$ is mechanical input power, $\mathrm{Pa}$ is accelerating power, $\mathrm{K}_{\mathrm{s} 1}$ is gain of PSS, $\mathrm{K}_{\mathrm{s} 2}$ is gain of inertial compensator, $\mathrm{K}_{\mathrm{s} 3}$ is gain of power combination, $\mathrm{K}$ is gain of automatic voltage regulator, $\mathrm{K}_{\mathrm{A}}$ is gain of excitation system's power, $K_{G}$ is gain of generator, $T_{A}$ is the time constant of power amplification compensator, $\mathrm{Td}^{\text {' }}$ is time constant of generator, $\mathrm{T}_{\mathrm{j}}$ is time constant of the total shaft, the $T_{r}$ is sampling time constant of generator's output voltage.

The accelerating power variation $\Delta \mathrm{P}_{\mathrm{a}}$ can be synthesized using signals of output power variation $\Delta \mathrm{P}_{\mathrm{e}}$ and speed variation $\Delta \omega, \Delta \mathrm{P}_{\mathrm{a}}$ is input signal of the threeorder lead-lag compensation compensator, $U_{\mathrm{pss}}$ can be got from the output of three-order lead-lag compensation compensator, which is added to the voltage reference value of the automatic voltage control loop. Physical concept of PSS2B model is clear, three-order lead-lag compensator has strong ability of phase correction and strong practicality.

\subsection{The phase compensation principle of the PSS2B model}

The phase relationship of each physical quantity in the PSS2B model is shown in Figure 2. Without compensation, as a result of the automatic voltage regulator control, the electromagnetic torque $\Delta \mathrm{T}_{\mathrm{e} 2}$ lags the power $(\Delta \delta)$, Component of the torque in the $\omega$-axis direction is negative, providing negative damping. PSS provides $\Delta U_{\text {PSS }}$ signal which is added to automatic voltage regulator by sampling and processing some input signals. The $\Delta U_{\text {PSS }}$ signal can provide positive damping through the lagging of the excitation system of

"Corresponding author: yf_zhu@e-mail.org 
electromagnetic torque $\Delta \mathrm{T}_{\mathrm{PSS}}$ in $\Delta \omega$ axis component is positive.

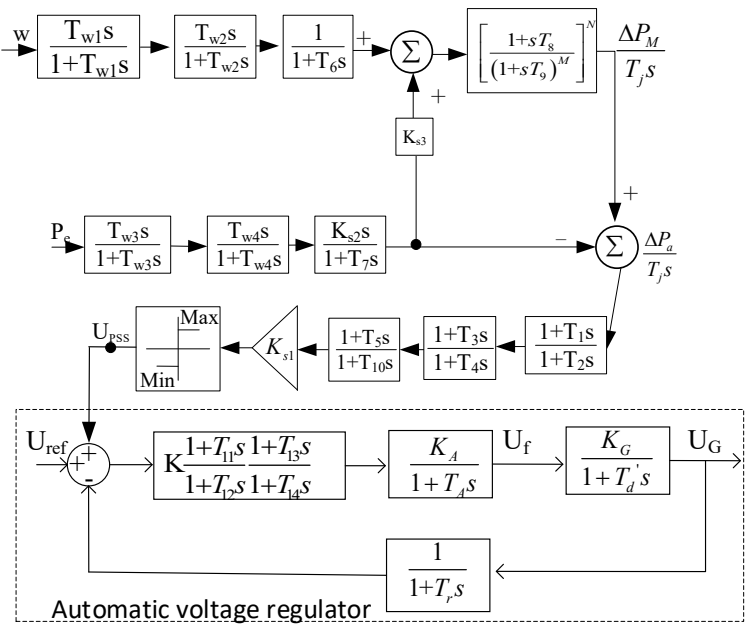

Figure 1 Transfer function of PSS2B model

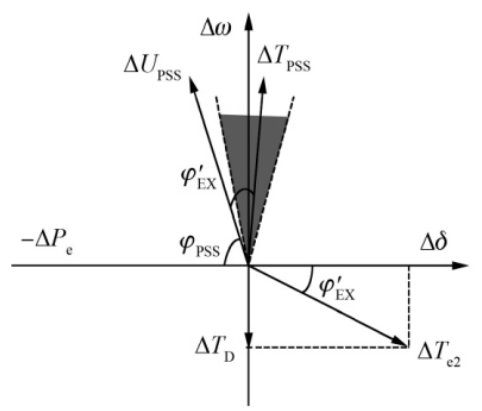

Figure 2 Phase diagram of the model

As shown in figure $2, \Delta \mathrm{T}_{\mathrm{PSS}}$ lags $-\Delta \mathrm{P}_{\mathrm{e}}$ axis $\varphi_{\omega}$, which contains lag angel of PSS itself and the inherent lag angle of the excitation system without compensation ${ }^{[5]}$. During PSS test, $\varphi_{E x}^{\prime}$ is obtained by frequency sweep, and the time constant of PSS lead-lag compensator is configured to meet the requirements ${ }^{[6]}$.

\section{Configuration method of PSS2B model's parameter}

The transfer function of PSS2B model as shown in figure 1, $\omega$ input branch is designed to suppress the Counter-modulation of PSS, during oscillation of active power, $\triangle \mathrm{Pm}$ equals zero, considering the configuration of PSS parameters is based on Pe axis, so lag angel of PSS itself can be expressed as,

$$
\begin{aligned}
& \varphi_{p s s}=\varphi\left(\frac{T_{w 3} s}{1+T_{w 3} s}\right)+\varphi\left(\frac{T_{w 4} s}{1+T_{w 4} s}\right)+\varphi\left(\frac{K_{s 2} s}{1+T_{7} s}\right) \\
& +\varphi\left(\frac{1+T_{1} s}{1+T_{2} s}\right)+\varphi\left(\frac{1+T_{3} s}{1+T_{4} s}\right)+\varphi\left(\frac{1+T_{5} s}{1+T_{6} s}\right)
\end{aligned}
$$

Where $T_{1} \sim T_{4}$ and $T_{7}$ are always set as 6 empirically, and the value of $K_{\mathrm{s} 2}$ is calculated from the inertia moment of total shaft and $\mathrm{T}_{7}$. The phase-frequency characteristic value after compensation has been stipulated clearly in DLT 1231-2018 "Guide for Setting
Test of Power System Stabilizer ", the lead-lag compensator time constant $\mathrm{T}_{1} \sim \mathrm{T}_{6}$ values should be configured during PSS test to meet the requirements. Therefore, how to configure the time constant quickly and effectively is a problem that needs to be studied and solved.

\subsection{Multivariate optimization function under constraints}

MATLAB fmincon() function can deal with the multivariate optimization problem, fmincon() function is structured as fmincon (FUN, X0, A, B, Aeq, Beq, LB, $\mathrm{UB}$ ), where FUN is the objective function and $\mathrm{X} 0$ is the initial value of the variable to be solved. The inequality constraints to be satisfied for variables is given by $\mathrm{A}$ and $\mathrm{B}(\mathrm{AX}<\mathrm{B})$, the equality constraints to be met for variables is given by Aeq and Beq (AeqX $=\mathrm{Beq})$, and the boundary conditions to be met for variables are given by $\mathrm{LB}$ and $\mathrm{UB}(\mathrm{LB}<\mathrm{X}<\mathrm{UB})$.

The optimization results are affected by the form of objective function directly. In this paper, the sum of difference squares between expected phase value and actual phase value at each frequency point is used as the optimization function. The expected phase angel $\varphi_{\omega 0}$ of $\Delta \mathrm{T}_{\mathrm{PSS}}$ lagging $-\Delta \mathrm{P}_{\mathrm{e}}$ at 20 discrete frequency points between $0.1 \sim 2.0 \mathrm{HZ}$ is set firstly, then the objective function myfun () is defined as sum of difference squares between expected phase value $\varphi_{\omega 0}$ and actual phase value $\varphi_{\omega}$,

$$
\text { myfun }=\sum_{f=0.1}^{2.0}\left[\varphi_{E X}^{\prime}(2 \pi f)+\varphi_{P S S}(2 \pi f)-\varphi_{\omega 0}(2 \pi f)\right]^{2}
$$

\subsection{Configuration method of PSS2B's lead-lag compensator time constant}

The configuration method of PSS2B lead-lag compensator time constant is shown in Figure 3. The first step is to set the desired phase angle $\varphi_{\omega 0}$ at each frequency point, Secondly the phase frequency value of the excitation system without compensation $\varphi_{E x}^{\prime}$ is obtained by frequency sweep and objective function myfun ( ) can be got according to (2), thirdly the optimal value of time constant can be obtained based on section 2.1 by using MATLAB fmincon() function. Fourthly time constants obtained in the previous step are substituted to calculate the actual phase value of each frequency point, whether the actual value meets the requirements is determined in the fifth step, the time constant value can be taken as the PSS setting value when requirements are met, otherwise, the second step is returned to modify the expected value of the phase angle at the frequency point where the requirement can not be met. 


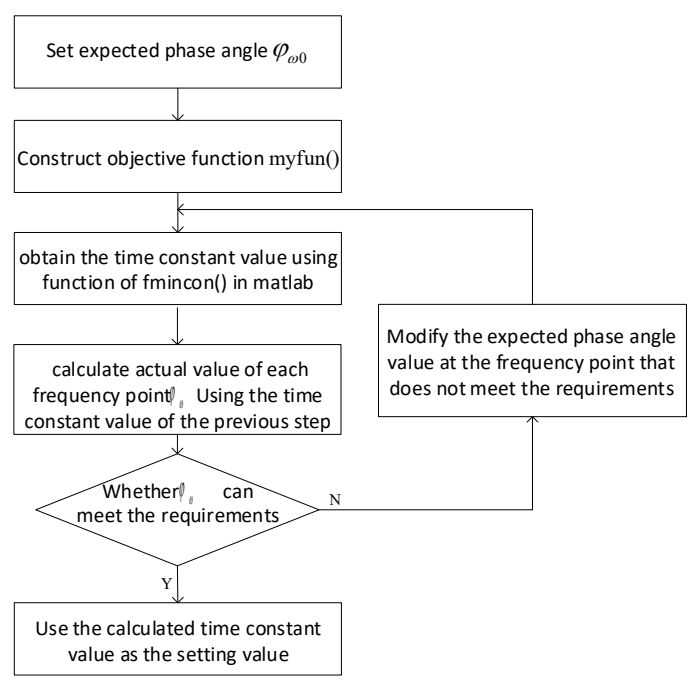

Figure 3 Configuration method of PSS model's lead-lag compensator time constant

\section{Test Verification}

The proposed configuration method of PSS lead-lag compensator time constant is verified in the PSS test of a $1000 \mathrm{MW}$ generator unit. The main parameters of the unit are shown in Table 1, the uncompensated phasefrequency characteristics of the excitation system are shown in Table 2. [6] stipulated that should be bigger than $60^{\circ}$ at $0.1 \sim 0.2 \mathrm{~Hz}$, so the expected angle can be set as $80^{\circ}$; within the range of $0.3 \sim 2.0 \mathrm{~Hz}$, should be larger than $70^{\circ}$ and smaller than $135^{\circ}$, so the expected value at each frequency point between $0.3 \sim 2.0 \mathrm{~Hz}$ can set as $100^{\circ}$. Using the method in Section 2, the following results can be obtained: $\mathrm{T}_{1}=0.1, \mathrm{~T}_{2}=0.01, \mathrm{~T}_{3}=0.30, \mathrm{~T}_{4}$ $=0.02$ (two-order lead-lag compensator is used), and the actual phase value after compensation is calculated. It is verified that the lead-lag angle of each frequency point meets the requirements, which can be used as the time constant of the PSS lead-lag compensator. The other parameters of PSS model are as follows: $\mathrm{T}_{1}=\mathrm{T}_{2}=\mathrm{T}_{3}=\mathrm{T}_{7}$ $=6, \mathrm{~T}_{4}=\mathrm{T}_{5}=\mathrm{T}_{6}=0, \mathrm{~T}_{8}=0.5, \mathrm{~T}_{9}=0.1, \mathrm{M}=5, \mathrm{~N}=1$, $\mathrm{Ks} 3=1$, all of which are empirical defaults. $K_{s 2}=\frac{1000 \cdot T_{7} \cdot S_{N}}{2.74 \cdot G D^{2} n^{2}}$, combined with table 1 , it can be calculated that $\mathrm{K}_{\mathrm{s} 2}=0.61$. Figure 4 shows the waveform of field voltage when $K_{s 1}=32$. As can be seen from figure 4 , the field voltage diverges to a certain extent, so the critical gain of Ks1 is 32 . Generally, $1 / 3 \sim 1 / 5$ of the critical gain is taken as the value of $\mathrm{Ks} 1^{[6]}$, so $\mathrm{Ks} 1$ is set as 8 .

Tab1 parameters of a $1000 \mathrm{Mw}$ generator unit

\begin{tabular}{|c|c|c|c|c|c|}
\hline parameter & unit & value & parameter & unit & value \\
\hline $\begin{array}{c}\text { Rated } \\
\text { capacity }\left(\mathrm{S}_{\mathrm{N}}\right)\end{array}$ & $\begin{array}{c}\text { MV } \\
\text { A }\end{array}$ & 1111 & $\begin{array}{c}\text { Rated } \\
\text { speed(n) }\end{array}$ & $\mathrm{r} / \mathrm{min}$ & 3000 \\
\hline $\begin{array}{c}\text { Power } \\
\text { factor }(\cos \varphi)\end{array}$ & $/$ & 0.9 & $\begin{array}{c}\text { Generator } \\
\text { flywheel } \\
\text { torque }\left(\mathrm{GD}^{2}\right)\end{array}$ & $\mathrm{t} \cdot \mathrm{m}^{2}$ & 68 \\
\hline $\begin{array}{c}\text { Generator } \\
\text { unit } \\
\text { voltage }\left(\mathrm{U}_{\mathrm{G}}\right)\end{array}$ & $\mathrm{Kv}$ & $\begin{array}{c}\text { Steam } \\
\text { turbine } \\
\text { flywheel } \\
\text { torque }\left(\mathrm{GD}^{2}\right)\end{array}$ & $\mathrm{t} \cdot \mathrm{m}^{2}$ & 373.8 \\
\hline
\end{tabular}

Tab2 Phase frequency characteristics with PSS compensation of a $1000 \mathrm{Mw}$ unit

\begin{tabular}{|c|c|c|c|}
\hline $\begin{array}{l}\text { frequency } \\
(\mathrm{HZ})\end{array}$ & $\begin{array}{c}\text { angle without } \\
\text { compensation } \varphi_{E x}^{\prime}\left({ }^{\circ}\right)\end{array}$ & $\begin{array}{l}\text { Angel } \\
\text { of } \\
\text { PSS } \\
\varphi_{p s s}\left({ }^{\circ}\right)\end{array}$ & $\begin{array}{c}\text { angle } \\
\text { with PSS } \\
\text { compens } \\
\text { ation } \\
\varphi_{\omega}\left(^{\circ}\right)\end{array}$ \\
\hline 0.1 & 26.7 & 46.7 & 73.4 \\
\hline 0.2 & 50.4 & 48.5 & 98.9 \\
\hline 0.3 & 63.2 & 41.9 & 105.1 \\
\hline 0.4 & 64.7 & 34.2 & 98.9 \\
\hline 0.5 & 70.3 & 26.8 & 97.1 \\
\hline 0.6 & 81.2 & 20.1 & 101.3 \\
\hline 0.7 & 82.1 & 14.1 & 96.2 \\
\hline 0.8 & 86.8 & 8.8 & 95.6 \\
\hline 0.9 & 85.5 & 4.1 & 89.6 \\
\hline 1.0 & 93.2 & 0.0 & 93.2 \\
\hline 1.1 & 113.5 & -3.8 & 109.7 \\
\hline 1.2 & 114.6 & -7.1 & 107.5 \\
\hline 1.3 & 115.7 & -10.0 & 105.7 \\
\hline 1.4 & 116.8 & -12.7 & 104.1 \\
\hline 1.5 & 118.3 & -15.1 & 103.2 \\
\hline 1.6 & 108.8 & -17.2 & 91.6 \\
\hline 1.7 & 115.6 & -19.1 & 96.5 \\
\hline 1.8 & 117.4 & -20.9 & 96.5 \\
\hline 1.9 & 121.4 & -22.4 & 99.0 \\
\hline 2.0 & 115.2 & -23.8 & 91.4 \\
\hline
\end{tabular}

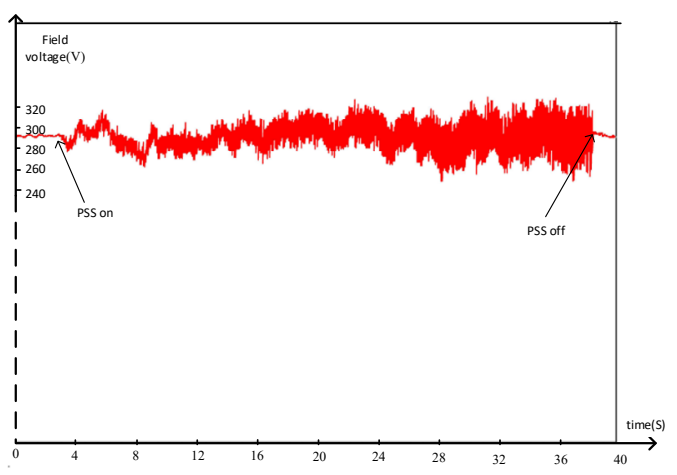

Figure 4 field voltage when Ks1 $=32$

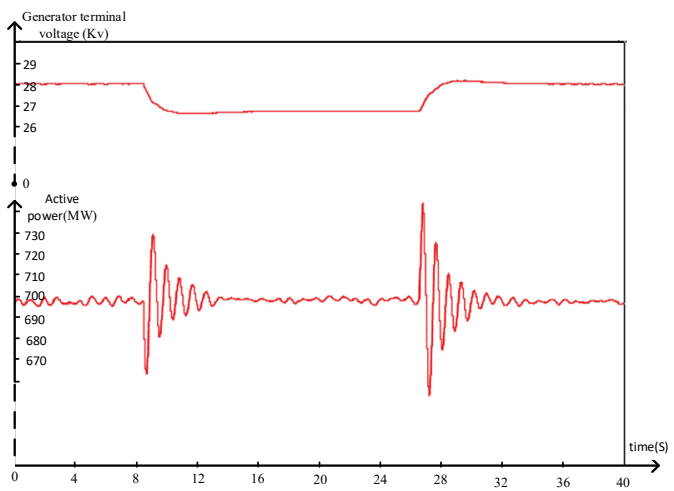

Figure 5 step test without PSS 


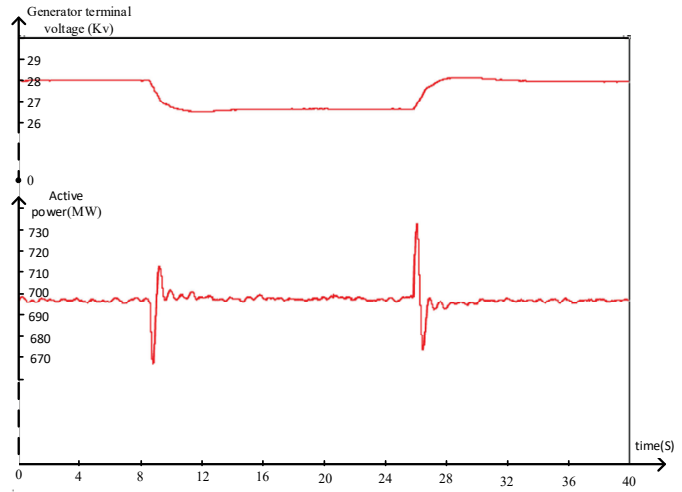

Figure 6 step test with PSS

As can be seen from figure 5 and figure 6 , under the same generator voltage change, the amplitude and times of active power oscillation are greatly reduced with PSS, indicating that the PSS lead-lag time constant configuration method proposed in this paper is effective.

\section{Acknowledgments}

The work described in this paper was fully supported by Science and Technology of State Grid Shandong Electric Power Company (NO. 520626190022).

\section{References}

1. YU Yixin, LI Peng. The impact of weak internection of bulk power grids to damping and dynamic stability of pwer systems[J]. Pro-ceedings of the Csee, 2005.

2. LIU Hui, YANG Yinping, TIAN Yunfeng, et, al. instances and mechanism analysis of force power oscillation in power systems[J] automation of electric power systems, 2012, 36(10):113-117.

3. JIA Hongjie, YU Yixin. The chaotic and bifurcaution phenomena considering power systems exciting limit and PSS.[J] automation of electric power systems, 2001, 25(1):11-14.

4. Dobson I, Zhang J, Greene S, et al. Is strong modal resonance a precursor to power system oscillations? [J]. IEEE Trans Circuits Syst, 2001, 48(3):340 - 349.

5. HUO Chengxiang, LIU Zenghuang, ZHU Fang. Theory and Field Practice of Phase Compensation on Generator Excitation System by Utilizing Power System Stabilizer[J]. Proceedings of the Csee, 2015, 35(12):2989-2997.

6. DLT1231-2013, Guide for Setting Test of Power System Stabilizer [S]. 